\title{
Multicarrier Orthogonal CDMA Signals for Quasi-Synchronous Communication Systems
}

\author{
Victor M. DaSilva, Student Member, IEEE, and Elvino S. Sousa, Member, IEEE
}

\begin{abstract}
We propose a multicarrier orthogonal CDMA signaling scheme for a multiple-access communication system, such as the reverse channel of a cellular network, as an alternative to the multi-user interference cancellation approach. The average variance of cross-correlations between sequences is used as a measure for sequence design. We search for sets of sequences that minimize the probability of symbol detection error, given that there is imperfect synchronization among the signals, that is, the signals are quasi-synchronous. Orthogonal sequences based on the Sylvester-type Hadamard matrices (Walsh functions) are shown to provide a significant improvement over the case where a Hadamard (orthogonal) matrix is chosen at random. Computer searches suggest that this set of codes is optimal with respect to the above measure. The issue of chip pulse shaping is investigated. Optimal pulses designed to minimize multiple-access interference in quasi-synchronous systems are obtained for various bandwidths and are shown to provide a large improvement over the raised cosine pulses. A multicarrier signaling scheme is introduced in order to reduce chip level synchronization offsets between the users.
\end{abstract}

\section{INTRODUCTION}

D IRECT sequence spread spectrum transmission has received considerable attention for applications in mobile and personal communication networks as a result of its potential to provide higher spectral efficiencies in comparison to conventional modulation techniques [1]-[3]. The potential increase in capacity over conventional modulation schemes is a result of the cellular structure of these systems and not a result of the efficiency of direct-sequence code-division multiple-access (DS CDMA) as a multiplexing scheme. In fact, in a one-cell system the efficiency of direct-sequence CDMA is considerably lower than that of TDMA or FDMA [4]. To improve on the capacity of a CDMA cellular network beyond the estimates in [1], more specialized signal processing techniques may be used in order to reduce the interference from terminals in the same cell. These techniques attempt to cancel the interference that arises as a result of nonzero crosscorrelation of the spreading codes [5]. Alternatively, we may attempt to synchronize the various transmissions at the chip level and to use orthogonal spreading codes, thus reducing the amount of multiple-access interference. This approach is

Manuscript received June 30, 1993; revised December 6, 1993. This paper was presented in part at the Second International Conference on Universal Personal Communications, Ottawa, October 1993.

The authors are with the Department of Electrical and Computer Engineering, University of Toronto, Canada.

This work was supported in part by the Natural Sciences and Engineering Research Council of Canada (NSERC) through a Postgraduate Scholarship, and in part by the Information Technology Research Center of Ontario, Canada (ITRC).

IEEE Log Number 9216745. used in the forward channel for the CDMA proposal discussed in [6]. However, its implementation in the reverse channel is more difficult, especially in the case of small chip periods. In an indoor environment the transmission delays are relatively small, hence, for low data rates and moderate processing gains, it may be feasible to maintain chip synchronization even in the reverse channel. This is the approach taken in the system proposed by Omura [3]. Assuming that there will be small errors in synchronization, orthogonal sequences with small cross-correlations for small synchronization offsets should be used. Some recent work that treats the problem of finding CDMA codes to reduce cross-interference is found in [7]-[9]. In [8] the $16 \times 16$ Sylvester matrix is found to minimize the mean square cross correlation for one chip offsets. A bandlimited quasi-synchronous CDMA system which uses raised cosine chip pulses is considered in [10].

We consider a one-cell system with one receiver base station and several mobile transmitters. While synchronization and orthogonality of signals can be maintained in the forward link, only quasi-synchronous operation can be maintained in the reverse link due to the differences in the transmitter clocks.

In this paper we analyze the performance of a quasisynchronous DS CDMA system and discuss a set of orthogonal codes that attempt to minimize the probability of symbol error. Optimal chip pulses of various bandwidths that minimize multiple-access interference under quasi-synchronous operation are obtained. As a means to reduce the synchronization offsets we introduce a multicarrier signaling scheme. With this scheme the chip period in each of the subcarriers is increased thus reducing the synchronization offsets by a factor equal to the number of subcarriers.

\section{SINGLE-CARRIER CDMA}

We consider a CDMA system with $N_{u}$ users. The received signal at the base station is

$$
r(t)=\sum_{i=1}^{N_{u}} A_{i} s_{i}\left(t-\tau_{i}\right) \cos \left(\omega_{0} t+\theta_{i}\right)+n(t)
$$

where $s_{i}(t)=\sum_{k=-\infty}^{\infty} d_{\lfloor k / N\rfloor}^{i} \tilde{c}_{k}^{i} h\left(t-k T_{c}\right)$ is the spreading code signal modulated by the data sequence $d_{l}^{i}= \pm 1$ and $\tilde{c}_{k}^{i}, A_{i}, \theta_{i}$, and $\tau_{i}$ are the spreading code sequence, amplitude, carrier phase, and delay of the $i^{\text {th }}$ signal, respectively, and $n(t)$ is the background noise process which has power spectral density $N_{0} / 2 .\lfloor x\rfloor$ denotes the largest integer less than or equal to $x, N$ is the processing gain, $h(t)$ is the chip pulse shape, $T_{c}$ is the chip period, and $T_{b}=N T_{c}$ is the symbol period. To 
simplify notation we define a new data-modulated chipping sequence $c_{k}=d_{\lfloor k / N\rfloor} \tilde{c}_{k}$.

We assume a correlator receiver. The output of the correlator due to the $k^{\text {th }}$ symbol is

$$
y \approx \pm \frac{A_{k} E_{s}}{2}+\psi+n
$$

where $E_{s}=\int_{0}^{T_{b}} s_{k}^{2}(t) d t, \psi$ is the multiple-access interference, and $\eta$ is a zero-mean Gaussian random variable with variance $N_{0} E_{s} / 4$.

To determine the probability of symbol error we need the distribution of $\psi$ which depends on the cross-correlation of the codes. Since we are assuming that the data symbols are equiprobable then by the central limit theorem $\psi$ approaches a Gaussian random variable as the number of interferers contributing to $\psi$ increases. We are interested in relatively large symbol error probabilities (e.g., $10^{-3}$ ). In this case and for a moderate processing gain it has been shown that $\psi$ can be approximated by a Gaussian distribution under quite general assumptions on the spreading codes (e.g., random codes, Gold codes). Under the Gaussian approximation the measure of interest is the variance of $\psi, \sigma_{\psi}^{2}$. We will use this measure to optimize the spreading code set. The variance of $\psi$ is

$$
\sigma_{\psi}^{2}=\mathcal{E}\left\{\psi^{2}\right\}=\sum_{i=1, i \neq k}^{N_{u}} \frac{A_{i}^{2}}{8} \mathcal{E}\left\{R_{s_{i} s_{k}}^{2}\left(\tau_{i}\right)\right\}
$$

where $\mathcal{E}\{\cdot\}$ denotes expectation over the data and code symbols and the relative delay $\tau_{i}$, and $R_{s_{i} s_{k}}=\int_{0}^{T_{b}} s_{i}(t-$ $\left.\tau_{i}\right) s_{k}(t) d t$.

The symbol error probability for the $k^{\text {th }}$ user is given by (e.g., see [11] for similar result)

$$
P_{e}=\frac{1}{2} \operatorname{crfc}\left(\sqrt{\frac{E_{k}}{N_{0}+\gamma \sum_{\substack{i=1 \\ i \neq k}}^{N_{u}} E_{i}}}\right)
$$

where $E_{i}=A_{i}^{2} \int_{0}^{T_{b}} s_{i}^{2}(t) d t / 2=A_{i}^{2} E_{s} / 2$ is the energy per symbol of the $i^{\text {th }}$ user and

$$
\gamma=\frac{\sum_{i=1, i \neq k}^{N_{u}} A_{i}^{2} \mathcal{E}\left\{R_{s_{i} s_{k}}^{2}\left(\tau_{i}\right)\right\}}{2 E_{s} \sum_{i=1, i \neq k}^{N_{u}} E_{i}}
$$

is the normalized variance of the multiple-access interference.

In this paper we study the performance of a quasisynchronous CDMA system where the reverse link transmissions from the different terminals arrive synchronized at the receiver base station to within a fraction of the chip period. We introduce the normalized offset $\Delta^{i}=\tau_{i} / T_{c}$ for the $i^{\text {th }}$ user. We refer to this variable generically as $\Delta(-1<\Delta<1)$. For the different users we model $\Delta$ as a random variable with probability density function $f_{\Delta}(\cdot)$, which is nonzero in $\left[-\Delta_{m}, \Delta_{m}\right], 0 \leq \Delta_{m}<1$.

For a synchronous system $\Delta_{m}=0$ and, assuming orthogonal codes and an appropriate chip pulse, the bit error probability is given by (4) with $\gamma=0$. For a quasi-synchronous system $\left(0<\Delta_{m}<1\right)$ with rectangular chip pulse, random spreading codes, and $\Delta$ uniformly distributed on $\left[-\Delta_{m}, \Delta_{m}\right]$ we have $\gamma=\left(1-\Delta_{m}+\frac{2}{3} \Delta_{m}^{2}\right) / N$; for $\Delta_{m}=1, \gamma=\frac{2}{3 N}$ and (4) becomes the well known result for asynchronous CDMA.

\section{A. Orthogonal CDMA Codes}

We assume that the spreading code period is equal to the symbol period and consider a rectangular chip pulse. The cross-correlation of the data-modulated code sequences for users $i$ and $k$ is

$$
R_{s_{i} s_{k}}\left(\Delta T_{c}\right)=T_{c} \sum_{l=0}^{N-1} c_{l}^{k}\left((1-|\Delta|) c_{l}^{i}+|\Delta| c_{l \pm 1}^{i}\right) .
$$

Given a specific set of orthogonal sequences, we can assign each sequence as the spreading code of a user. The error probability for a given user will depend on the set of active users (up to a maximum equal to the number of orthogonal sequences), and on the chip offsets for the interfering users relative to the user of interest. In previous research the criterion for sequence set design has been based on the worst case cross correlations (e.g., Gold codes). This criterion is applicable in the case of high SNR. The current trend, especially in mobile communications, is to use forward error correction codes and to design the system to operate at a relatively large symbol error rate (e.g., $10^{-3}$ and higher). In this case a criterion that is based on the mean square cross correlation value is more appropriate.

Taking all users into account, we would like to use a set of sequences where the average of the squares of the crosscorrelations over all pairs of sequences is minimized. If it turns out that the squared cross-correlations for the different users vary considerably with respect to the overall average then the performance of the different users can be made equal by having each mobile change its code cyclically over the whole set of codes such that no two mobiles use the same code simultaneously.

To minimize cross-interference, we seek an orthogonal set that minimizes $\mathcal{E}\left\{R_{s_{i} s_{k}}^{2}\left(\Delta T_{c}\right)\right\}$, where the expectation is taken over the random variable $\Delta$ and over the data-modulated spreading sequences from the orthogonal set (i.e., average over all pairs of codes in the set). We write

$$
\mathcal{E}\left\{R_{s_{i} s_{k}}^{2}\left(\Delta T_{c}\right)\right\}=T_{c}^{2} \mathcal{E}\left(\Delta^{2}\right) \mu_{\text {cor }}(N)
$$

where

$$
\mu_{\mathrm{cor}}(N)=\mathcal{E}\left\{\left(\sum_{l=0}^{N-1} c_{l}^{k} c_{l+1}^{i}\right)^{2}\right\}
$$

To minimize (7) we need to minimize $\mu_{\text {cor }}(N)$. Given an $N \times N$ orthogonal matrix $C=\left[c_{j}^{i}\right]$ with $c_{j}^{i} \in\{-1,1\}$, (a Hadamard matrix) the rows are assigned as code sequences to a maximum of $N$ users. The average of the squared crosscorrelations of the sequences, under a one chip offset, is

$$
\mu_{\text {cor }}(N)=\frac{1}{2}\left(\mu_{\text {cor }+}+\mu_{\text {cor }-}\right)
$$

where

$$
\mu_{\mathrm{cor}+}=\frac{1}{N(N-1)} \sum_{\substack{k=1 \\ N}}^{N} \sum_{\substack{i=1 \\ i \neq k}}^{N}\left(\sum_{j=0}^{N-1} c_{j}^{k} c_{j+1}^{i}\right)^{2}
$$


and where $c_{N}^{i}=c_{0}^{i}$. We define $\mu_{\text {cor- }}$ the same as $\mu_{\text {cor+ }+}$ except that we set $c_{N}^{i}=-c_{0}^{i}$. Our problem is to find the Hadamard matrix $C$ which minimizes (9).

For the variance of the multiple-access interference $\psi$ to be minimized, (7) suggests that $\mu_{\mathrm{cor}}(N)$ should be a minimum, regardless of the distribution for $\Delta$. If we take $\Delta$ to be uniformly distributed on $\left[-\Delta_{m}, \Delta_{m}\right]$ the symbol error probability of the resulting quasi-synchronous CDMA system can then be obtained by substituting

$$
\gamma=\frac{\Delta_{m}^{2} \mu_{\text {cor }}(N)}{3 N^{2}}
$$

into (4), hence $P_{e}$ is minimized when $\mu_{\text {cor }}(N)$ is minimized.

The bit error probability given by (4) is not the average bit error rate for all users. We interpret (4) as the bit error rate associated with a typical user subjected to an averaged cross-interference noise.

We wish to find Hadamard matrices $C$ which minimize $\mu_{\text {cor }}(N)$. We restrict the search to the case $N=2^{n}$. We note that interchanging and negating the rows of a Hadamard matrix produces an equivalent Hadamard matrix with the same $\mu_{\text {cor }}(N)$ value; nonequivalent matrices are referred to as distinct. If $N$ is small, then it is a simple task to find all distinct $N \times N$ Hadamard matrices and to compute $\mu_{\text {cor }}(N)$ for each. However, for large $N$ the set of distinct Hadamard matrices is unknown [12]. We have performed a search for Hadamard matrices with low values of $\mu_{\text {cor }}(N)$. In all cases of $N$ considered, the best Hadamard matrices found were equivalent to the $N \times N$ Sylvester-type Hadamard matrix, which is defined recursively as follows

$$
\mathbf{H}_{N}=\left[\begin{array}{rr}
\mathbf{H}_{N / 2} & \mathbf{H}_{N / 2} \\
\mathbf{H}_{N / 2} & -\mathbf{H}_{N / 2}
\end{array}\right]
$$

where $\mathbf{H}_{1}=1$.

For the case $N=4$, there exist two distinct Hadamard matrices, and the smallest correlation is $\mu_{\text {cor }}^{*}=10 / 3$. For the case $N=8$ an exhaustive search over all Hadamard matrices was performed and we obtained $\mu_{\text {cor }}^{*}=6$ corresponding to the $8 \times 8$ Sylvester matrix. For higher values of $N$, Hadamard matrixes were generated randomly by interchanging and negating the columns of a given Hadamard matrix. This process yields a large portion of the distinct matrices but not all of them in general [12, p. 48]. We generated 100000 matrices for $N=16,100000$ for $N=32,10000$ for $N=64,4000$ for $N=128$, and 500 for $N=256$. The histograms for the $\mu_{\text {cor }}$ values for two cases of $N$ are shown in Figs. 1-2. In all cases the $\mu_{\text {cor }}$ values have a distribution concentrated around $N$. The spread of the distributions decreases, relative to $N$, as $N$ increases except for an isolated point (corresponding to the Sylvester matrix) at a value of approximately $\frac{2}{3} N$. The exact value, derived in the Appendix, is as follows

$$
\mu_{\text {cor }}(N)=\frac{2}{3}(N+1)
$$

\section{B. The Gaussian Approximation}

Given that the spreading sequences are the rows of a Hadamard matrix, it is of interest to verify the Gaussian approximation in computing the probability of bit error (4)

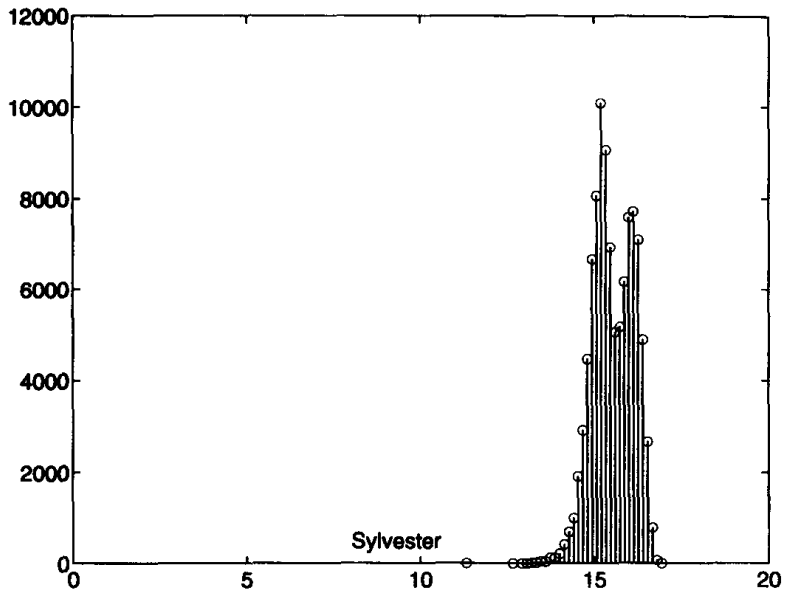

Fig. 1. Number of occurrences of $\mu_{\text {cor }}$ versus $\mu_{\text {cor }}$ for the case $N=16$. 100,000 randomly generated orthogonal matrices were investigated

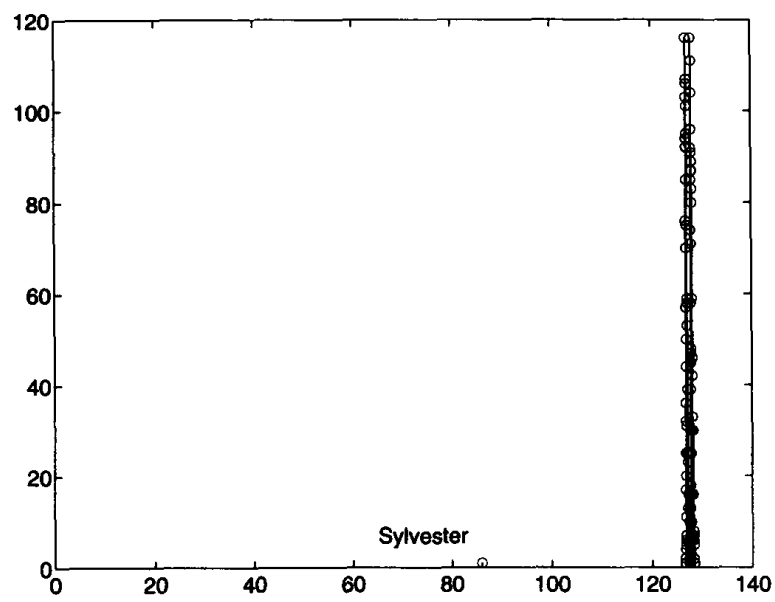

Fig. 2. Number of occurrences of $\mu_{\text {cor }}$ versus $\mu_{\text {cor }}$ for the case $N=128$. 4000 randomly generated orthogonal matrices were investigated.

which was obtained under the assumption of random sequences. We have performed an exact computation for the case $N_{u}=N=8$ assuming that $\Delta$ is uniformly distributed on $\left[-\Delta_{m}, \Delta_{m}\right]$, the signals are baseband signals, and that all users have the same bit energy $E_{i}=E_{b}$. It turns out that the probability of error is code dependent. There are four classes of spreading codes, with two codes per class, and each class has a different probability of error as shown in Fig. 3, for the case $\Delta_{m}=.5$. In Fig. $4(\mathrm{~b})$, we show the average probability of bit error over the four classes of codes. By using the Gaussian approximation for each class of codes we obtain error probability expressions given by (4) with four corresponding $\gamma$ 's; Fig. 4(a) shows the average of these. The curve obtained via the Gaussian approximation is pessimistic at an error probability of $10^{-3}$ by approximately $E_{b} / N_{0}=1.5$ $\mathrm{dB}$. The approximation should be considerably better for larger values of $N$. We can modify the signaling scheme by having each user change the spreading code after each bit (cyclically 


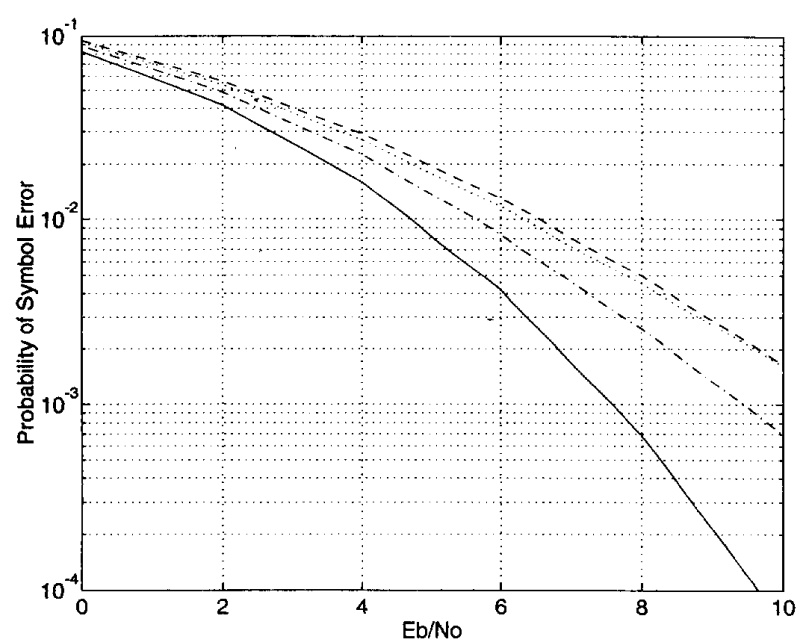

Fig. 3. Exact probability of symbol error versus $\frac{E_{b}}{N_{0}}$ for a system using the $8 \times 8$ Sylvester matrix, $N_{u}=8, \Delta_{m}=.5$.

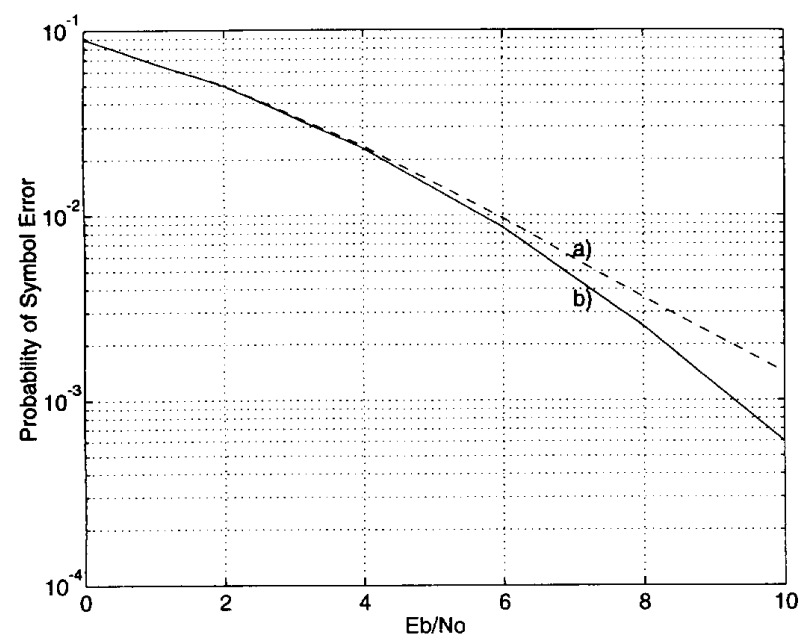

Fig. 4. Probability of symbol error versus $\frac{E_{b}}{N_{0}}$ averaged over the users for a system using the $8 \times 8$ Sylvester matrix, $N_{u}=8, \Delta_{m}=.5$ : (a) Gaussian approximation, (b) exact computation.

through all codes) so that all users would have the performance shown in Fig. 4.

Assuming large $N$, and substituting $\mu_{\text {cor }}(N)=2 N / 3$ into (11), we obtain $\gamma=2 \Delta_{m}^{2} /(9 N)$ for the $N \times N$ Sylvester matrix. For a typical non-Sylvester matrix $\mu_{\text {cor }} \approx N$ and $\gamma=\Delta_{m}^{2} /(3 N)$; thus the multiple access interference is reduced by a factor of $3 / 2$ in the case of a Sylvester matrix. Fig. 5 and Fig. 6 show plots of the bit error probability given by (4) (assuming $E_{i}=E_{k}=E_{b}$ ), for typical users (i.e., using the average squared cross correlation values), for a) random sequences, b) typical non-Sylvester orthogonal sequences, and c) Sylvester sequences.

The improvement in performance of the Sylvester sequences over typical non-Sylvester sequences becomes more noticeable as $\Delta_{m}$ increases. The difference in the maximum number of

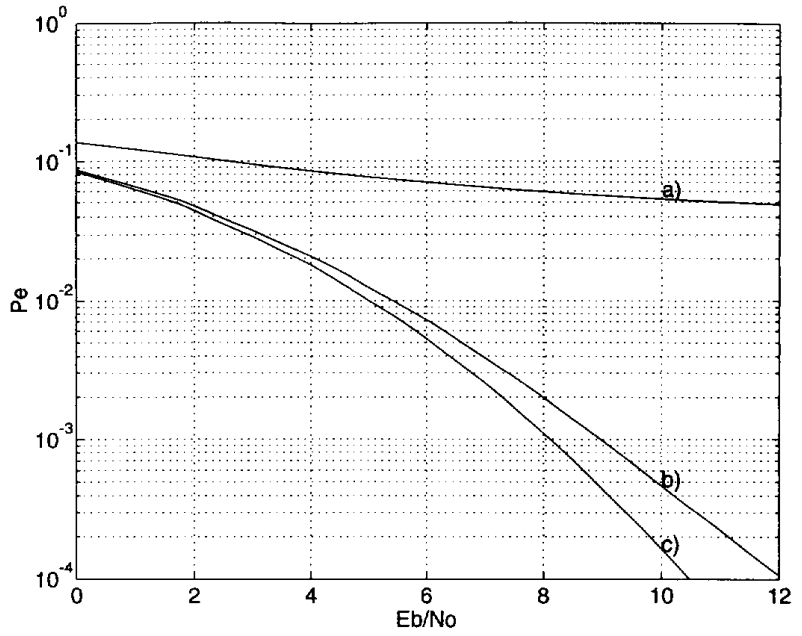

Fig. 5. Error probability $P_{\epsilon}$ versus $\frac{E_{b}}{N_{0}}$ for a typical user in the case of (a) nonorthogonal sequences, (b) non-Sylvester orthogonal sequences, and (c) Sylvester sequences. $N_{u}=N=128, \Delta_{m}=.5$.

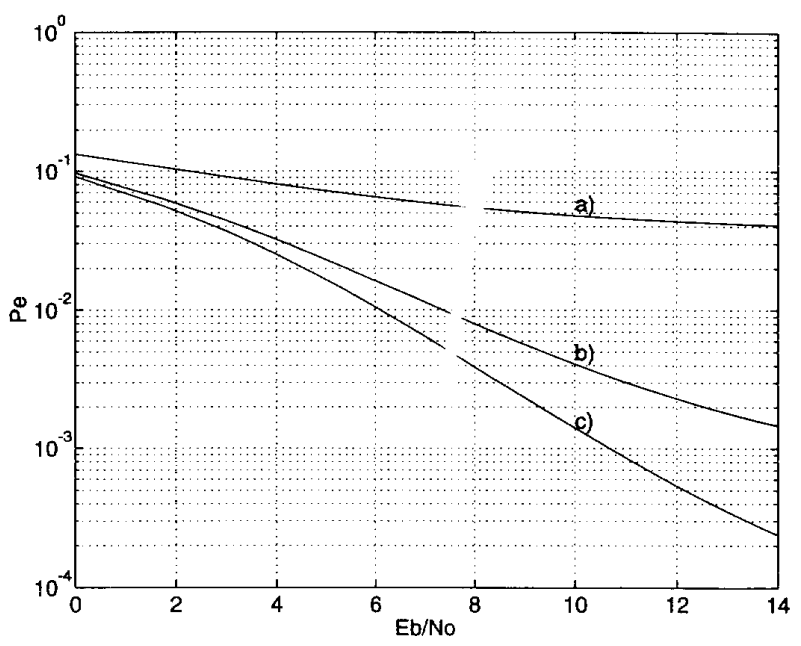

Fig. 6. Error probability $P_{e}$ versus $\frac{E_{b}}{N_{0}}$ for a typical user in the case of (a) nonorthogonal sequences, (b) non-Sylvester orthogonal sequences, and (c) Sylvester sequences. $N_{u}=N=128, \Delta_{m}=.75$.

simultaneous users for Sylvester and non-Sylvester Hadamard sequences, for a given probability of error, depends on the level of background noise $N_{0}$. Given a maximum operating probability of error, (4) may be solved for the maximum number of simultaneous users. Quasi-synchronous CDMA systems based on Sylvester sequences and non-Sylvester Hadamard sequences may then be compared in terms of capacity, whereas Figs. 5 and 6 compare them in terms of SNR.

\section{General Chip Pulse Shape}

We now consider the case of a nonrectangular chip pulse shape. Let $g(t)=\int_{-\infty}^{\infty} h(u) h(u-t) d u$. With perfect synchronization, orthogonality of signals can be maintained by using orthogonal spreading codes and a chip pulse which satisfies 
the Nyquist I criterion,

$$
g\left(l T_{\mathrm{c}}\right)=\left\{\begin{array}{cl}
E_{h}, & l=0 \\
0, & l= \pm 1, \pm 2, \pm 3 \cdots
\end{array}\right.
$$

Let us consider the case where the users are quasisynchronous. The pulse that minimizes the cross-interference will not necessarily satisfy (14). Consider a pair of baseband CDMA signals, $s_{i}(t+\tau)=\sum_{m=-\infty}^{\infty} c_{m}^{i} h\left(t+\tau-m T_{\mathrm{c}}\right)$ and $s_{k}(t)=\sum_{n=-\infty}^{\infty} c_{n}^{k} h\left(t-n T_{c}\right)$, where $-T_{c}<\tau<T_{c}$. The design problem is as follows: for a given chip pulse bandwidth and pdf for the synchronization offset, find the optimum set of orthogonal sequences and optimum chip pulse shape. The cost function to be minimized is the average of the squared cross correlations for all pairs of signals. The cross correlation between two signals is

$$
\begin{aligned}
\psi & =\int_{0}^{T_{b}} s_{i}(t+\tau) s_{k}(t) d t \\
& \approx \sum_{n=0}^{N-1} \sum_{\substack{m=-\infty \\
m \neq n}}^{\infty} c_{m}^{i} c_{n}^{k} \int_{-\infty}^{\infty} h\left(t+\tau-m T_{c}\right) h\left(t-n T_{c}\right) d t \\
& =\sum_{\substack{l=-\infty \\
l \neq 0}}^{\infty} \sum_{n=0}^{N-1} c_{n}^{k} c_{n+l}^{i} g\left(l T_{c}-\tau\right)
\end{aligned}
$$

where in the second step we have assumed that $h(t)$ contains most of its energy in the interval $t \in\left[0, T_{c}\right]$, that $N$ is large, and that the codes are orthogonal.

The variance of $\psi$ may be computed as follows,

$$
\sigma_{\psi}^{2}=\mathcal{E}\left\{\psi^{2}\right\} \approx \sum_{\substack{l=-\infty \\ l \neq 0}}^{\infty} \mathcal{E}\left\{\left(\sum_{n=0}^{N-1} c_{n}^{k} c_{n+l}^{i}\right)^{2}\right\} \mathcal{E}\left\{g^{2}\left(l T_{c}-\tau\right)\right\}
$$

where $c_{N+j}^{i}= \pm c_{j}^{i}$ depending on whether or not consecutive data symbols change sign.

Define

$$
\mu_{l}=\frac{1}{2}\left(\mu_{l+}+\mu_{l-}\right)
$$

where

$$
\begin{aligned}
\mu_{l+} & =\mathcal{E}\left\{\left(\sum_{n=0}^{N-1} c_{n}^{k} c_{n+l}^{i}\right)^{2}\right\} \\
& =\frac{1}{N(N-1)} \sum_{\substack { k=1 \\
\begin{subarray}{c}{i=1 \\
i \neq k{ k = 1 \\
\begin{subarray} { c } { i = 1 \\
i \neq k } }\end{subarray}}^{N}\left(\sum_{j=0}^{N-1} c_{j}^{k} c_{j+l}^{i}\right)^{2}
\end{aligned}
$$

and $c_{N+j}^{i}=c_{j}^{i}$, and $\mu_{l-}$ is defined similarly except that $c_{N+j}^{i}=-c_{j}^{i}$. Note that (18) with $l=1$ is $\mu_{\text {cor }}(N)$ in (9). We note that $\mu_{l}=\mu_{-l}$ since the expectation is taken over all pairs of rows of an orthogonal matrix. Since $g(\cdot)$ and $f_{\Delta}(\cdot)$ are even functions, we rewrite $\sigma_{\psi}^{2}$ as

$$
\sigma_{\psi}^{2}=2 \sum_{l=1}^{\infty} \mu_{l} \mathcal{E}\left\{g^{2}\left(l T_{c}-\tau\right)\right\}
$$

For a given bandwidth of $h(t)$ and a given processing gain $N=2^{n}$, we wish to find the optimal set of orthogonal sequences and the optimal pulse shape such that $(20)$ is minimized. Since the function $g^{2}(t)$ decays rapidly with increasing $t$ we expect that the first few terms in the sum (20) dominate; hence we choose an orthogonal set of sequences with low $\mu_{l}$ for small $l$. For the Sylvester sequences $\mu_{l}=2 N / 3$ for $l=1,2$, compared to $\mu_{l}=N$ for typical orthogonal sequences, hence we conclude that these are close to optimal and choose them. We have thus decoupled the problems for finding the optimum sequences and the optimum pulse shape.

To solve for the optimum $g(t)$ we minimize (20) subject to a constraint on the bandwidth and the energy of the pulse $\int_{-\infty}^{\infty} h^{2}(t) d t=E_{h}=g(0)$. For small synchronization offsets we present an analytical solution. For larger offsets, we solve the problem using a numerical approach. Note that the optimum pulse will not necessarily be a Nyquist I pulse.

For small synchronization offsets we can use a two-term Taylor series expansion of $g(t)$ at $l T_{c}$ to obtain $g\left(l T_{c}-\tau\right) \approx$ $g\left(l T_{c}\right)-\tau g^{\prime}\left(l T_{c}\right)$. Squaring, noting that the mean of $\tau$ is 0 , computing the expectation over the random variable $\tau$, and summing over $l$ we express the cost function (20) as

$$
\sigma_{\psi}^{2}=2 \sum_{l=1}^{\infty} \mu_{l}\left(g^{2}\left(l T_{C}\right)+\left[g^{\prime}\left(l T_{c}\right)\right]^{2} \sigma_{\tau}^{2}\right)
$$

where $\sigma_{\tau}^{2}$ is the variance of $\tau$. If we approximate the $\mu_{l}$ 's by a constant then we obtain a cost function that is similar to a cost function obtained in [13] for an unrelated problem. From [13] the following pulse minimizes (21), if we assume that $\mu_{l}$ is a constant and that $\sigma_{\tau}^{2}$ is small:

$$
G(f) \approx \begin{cases}c, & |f|<\frac{1-\beta}{2 T_{c}} \\ c\left(1-|f| T_{c}\right), & \frac{1-\beta}{2 T_{c}} \leq|f| \leq \frac{1+\beta}{2 T_{c}} \\ 0, & |f|>\frac{1+\beta}{2 T_{c}}\end{cases}
$$

where $c$ is a constant such that $\int_{-\infty}^{\infty} G(f) d f=E_{h}$, and $\beta$ is the fractional excess bandwidth.

Even if we make the approximation that $\mu_{l}$ is a constant, for larger synchronization offsets the above Taylor series method cannot be used. In this case since $g(t)$ is band-limited we expand it as a sum of weighted $\operatorname{sinc}(\cdot)$ functions

$$
g(t)=\sum_{k=0}^{L} x_{k} \phi_{k}(t)
$$

where

$$
\phi_{k}(t)=\operatorname{sinc}\left(\frac{(1+\beta) t}{T_{c}}-k\right)+\operatorname{sinc}\left(\frac{(1+\beta) t}{T_{c}}+k\right)
$$

and $\operatorname{sinc}(x)=\sin (\pi x) /(\pi x)$. The bandwidth of $g(t)$ is $\frac{1+\beta}{2 T_{c}}$. Substituting (23) into (20), and defining $f_{i j l}=\mathcal{E}\left\{\phi_{i}(\tau-\right.$ $\left.\left.l T_{c}\right) \phi_{j}\left(\tau-l T_{c}\right)\right\}$, we obtain the functional

$$
F=\sigma_{\psi}^{2}=2 \sum_{l=1}^{\infty} \mu_{l}\left(\sum_{i=0}^{L} \sum_{j=0}^{L} x_{i} x_{j} f_{i j l}\right)
$$

to be minimized subject to the constraint $\sum_{k=0}^{L} x_{k} \phi_{k}(0)=$ $E_{h}$. 


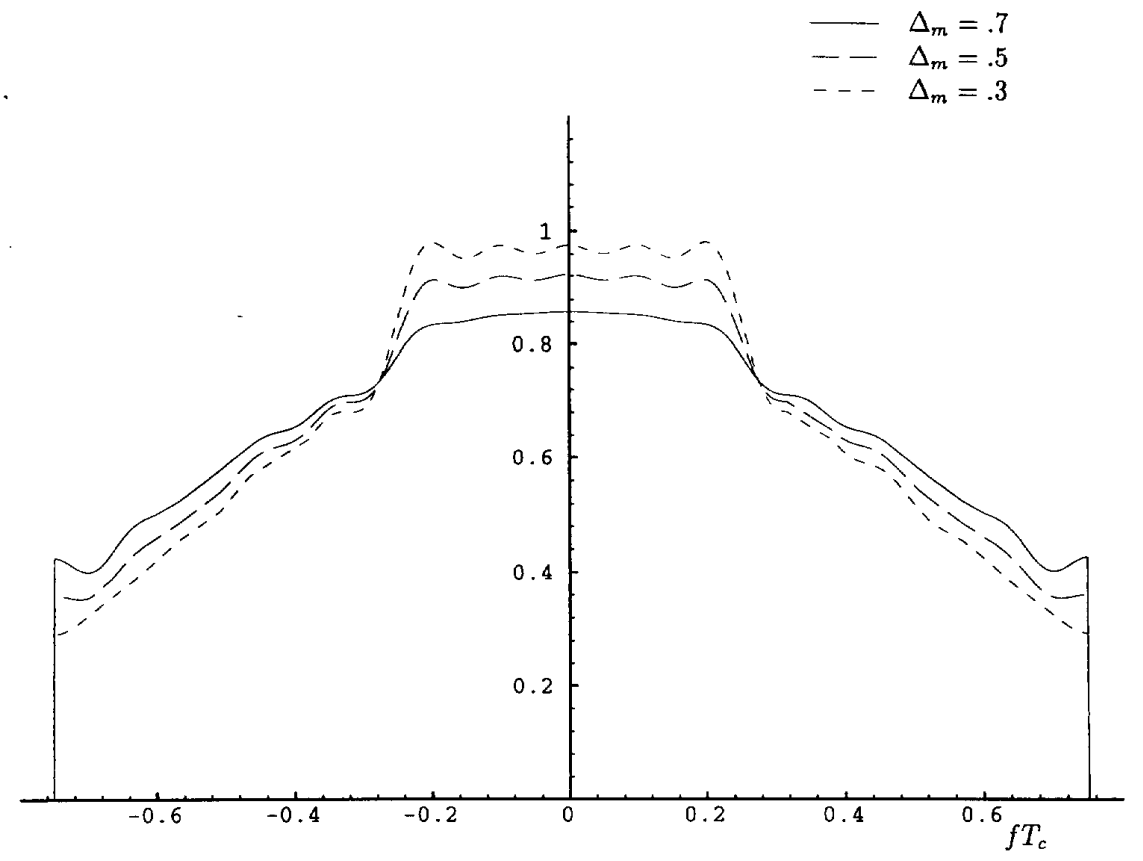

Fig. 7. Optimum chip pulse $\frac{G(f)}{E_{h} T_{c}}$, for $\beta=.5$.

The solution, based on Lagrange multipliers, reduces to the solution for the linear system

$$
\mathbf{A x}=\mathbf{b}
$$

where the variables are defined at the bottom of the page, and $\lambda$ is a Lagrange multiplier.

\section{Optimum Chip Pulse Shape}

If the system attempts to maintain the signals synchronized we expect that the probability density function of $\Delta$ should have a maximum at zero. As an example, we solve the optimal chip pulse problem for the following density function.

$$
f_{\Delta}(\Delta)= \begin{cases}\left(1-\frac{|\Delta|}{\Delta_{m}}\right) \frac{1}{\Delta_{m}}, & |\Delta| \leq \Delta_{m} \\ 0, & |\Delta|>\Delta_{m}\end{cases}
$$

We discuss solutions to (26) for various values of $\Delta_{m}$ and fractional excess bandwidths $\beta$. In the frequency domain, the optimal pulse has the form

$$
G(f)= \begin{cases}\frac{2 T_{c}}{1+\beta} \sum_{k=0}^{L} x_{k} \cos \left(\frac{T_{c} 2 \pi k f}{1+\beta}\right), & |f| \leq \frac{1+\beta}{2 T_{c}} \\ 0, & |f|>\frac{1+\beta}{2 T_{c}}\end{cases}
$$

Figs. 7-8 show $G(f)$ for two different bandwidths, and for each bandwidth, the three cases $\Delta_{m}=.7, .5$, and .3 . We have used $L=15$. Also, note that the results for $\Delta_{m}=.3$ and all the $\beta$ values resemble the analytical solution given by (22). The values used for $\mu_{l}$ correspond to the limiting values (large $N$ ) for Sylvester sequences, and are as follows,

$\left(\mu_{1}, \mu_{2}, \mu_{3}, \mu_{4}, \mu_{5}, \mu_{6}, \mu_{7}, \mu_{8}, \mu_{9}\right)$

$$
\begin{aligned}
& =N\left(\frac{2}{3}, \frac{2}{3}, \frac{5}{6}, \frac{2}{3}, \frac{7}{8}, \frac{5}{6}, \frac{7}{8}, \frac{2}{3}, \frac{7}{8}\right) \\
\mu_{l} & =N, \text { for } l \geq 10
\end{aligned}
$$

The value for $l \geq 10$ is an approximation.

In $(26)$

$$
\begin{aligned}
& \mathbf{A}=\left[\begin{array}{cccccc}
\sum_{l=1}^{\infty} \mu_{l} f_{00 l} & \sum_{l=1}^{\infty} \mu_{l} f_{01 l} & \sum_{l=1}^{\infty} \mu_{l} f_{02 l} & \cdots & \sum_{l=1}^{\infty} \mu_{l} f_{0 L l} & \frac{1}{2} \\
\sum_{l=1}^{\infty} \mu_{l} f_{10 l} & \sum_{l=1}^{\infty} \mu_{l} f_{11 l} & \sum_{l=1}^{\infty} \mu_{l} f_{12 l} & \cdots & \sum_{l=1}^{\infty} \mu_{l} f_{1 L l} & 0 \\
\sum_{l=1}^{\infty} \mu_{l} f_{20 l} & \sum_{l=1}^{\infty} \mu_{l} f_{21 l} & \sum_{l=1}^{\infty} \mu_{l} f_{22 l} & \cdots & \sum_{l=1}^{\infty} \mu_{l} f_{2 L l} & 0 \\
\vdots & & & & & \\
\sum_{l=1}^{\infty} \mu_{l} f_{L 0 l} & \sum_{l=1}^{\infty} \mu_{l} f_{L 1 l} & \sum_{l=1}^{\infty} \mu_{l} f_{L 2 l} & \cdots & \sum_{l=1}^{\infty} \mu_{l} f_{L L l} & 0 \\
\frac{1}{2} & 0 & 0 & \cdots & 0 & 0
\end{array}\right] \\
& \mathbf{x}^{T}=\left[x_{0}, x_{1}, x_{2}, x_{3}, \ldots, x_{L}, \lambda\right], \quad \mathbf{b}^{T}=\left[0,0,0,0, \ldots, 0, \frac{E_{h}}{4}\right] .
\end{aligned}
$$




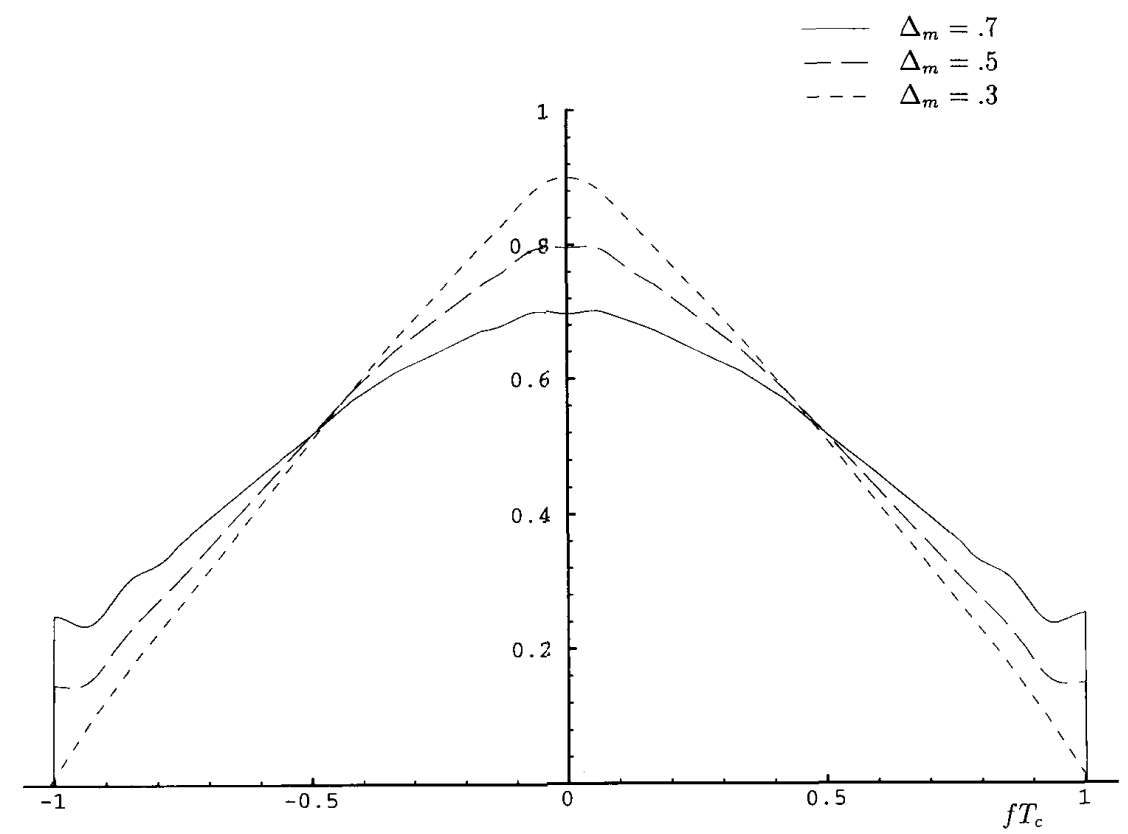

Fig. 8. Optimum chip pulse $\frac{G(f)}{E_{h} T_{c}}$, for $\beta=1$.

TABLE I

NORMALIZED COST $\frac{F}{E_{h}^{2} N}$

\begin{tabular}{|c|c|c|c|}
\hline $\begin{array}{c}\text { Fractional } \\
\text { Excess bandwidth }(\beta)\end{array}$ & $\begin{array}{c}\text { Normalized cost for } \\
\text { sq. root R.C. pulse }\end{array}$ & $\begin{array}{c}\text { Normalized cost for } \\
\text { optimal pulse }\end{array}$ & $\begin{array}{c}\text { Improvement } \\
\text { factor }\end{array}$ \\
\hline 0 & .141 & .137 & 1.03 \\
\hline .25 & .110 & .072 & 1.5 \\
\hline .50 & .082 & .040 & 2.1 \\
\hline .67 & .067 & .027 & 2.5 \\
\hline .75 & .061 & .022 & 2.8 \\
\hline 1 & .045 & .013 & 3.5 \\
\hline
\end{tabular}

We now compare the performance of the optimal chip pulses versus the class of square root raised cosine pulses $(30)$, see below. Table I lists the normalized costs $\sigma_{\psi}^{2} /\left(E_{h}^{2} N\right)$ associated with the optimal pulses and the square root raised cosine pulses for six different bandwidths for the case $\Delta_{m}=.7$. We note that the improvement of the optimal pulse over the raised cosine pulse becomes significant as the bandwidth is increased.

We now consider the implications of Table I on the performance. Considering a CDMA system with $N_{u}$ users, we can show that (5) reduces to

$$
\gamma=\frac{F \sum_{\substack{i=1 \\ i \neq k}}^{N_{u}} A_{i}^{2}}{2 E_{s} \sum_{\substack{i=1 \\ i \neq k}}^{N_{u}} E_{i}} .
$$

Using the approximation $E_{i}=A_{i}^{2} \int_{0}^{T_{b}} s_{i}^{2}(t) d t / 2 \approx$
$A_{i}^{2} N E_{h} / 2$, that is, the bit energy is $N$ times the chip energy, (31) reduces to,

$$
\gamma=\frac{F}{N^{2} E_{h}^{2}}
$$

which is substituted into (4) to obtain the probability of error. In Fig. 9 we have plotted the probability of symbol error given by (4) for different pulse shapes and code sequences assuming $\Delta_{m}=.7$ and $\beta=.5$. Three combinations are considered: 1 ) randomly chosen orthogonal sequences and square root raised cosine chip pulses, 2) Sylvester sequences and square root raised cosine pulses, and 3) Sylvester sequences and optimal chip pulses. The b) and c) curves are computed using the two corresponding $\gamma$ 's computed from Table I. The a) curve is computed assuming $\mu_{l}=N, l \geq 1$. At a probability of error of $10^{-3}$ there is a difference of approximately $3 \mathrm{~dB}$ between the curve corresponding to the Sylvester sequences and optimum chip pulse shape and that corresponding to a typical set of orthogonal sequences with square root raised cosine chip pulse shape.

\section{MUlti-CARRIER CDMA}

To reduce the probability of bit error the synchronization offsets should be a small fraction of the chip period. Increasing the chip period would make it easier to synchronize the signals.

$$
G(f)=\left\{\begin{array}{lc}
E_{h} T_{\mathrm{c}}, & |f|<\frac{1-\beta}{2 T_{c}} \\
\frac{E_{h} T_{c}}{2}\left(1+\cos \left[\frac{\pi T_{c}}{\beta}\left(|f|-\frac{1-\beta}{2 T_{c}}\right)\right]\right), & \frac{1-\beta}{2 T_{c}} \leq|f| \leq \frac{1+\beta}{2 T_{c}} \\
0, & |f|>\frac{1+\beta}{2 T_{c}}
\end{array}\right.
$$




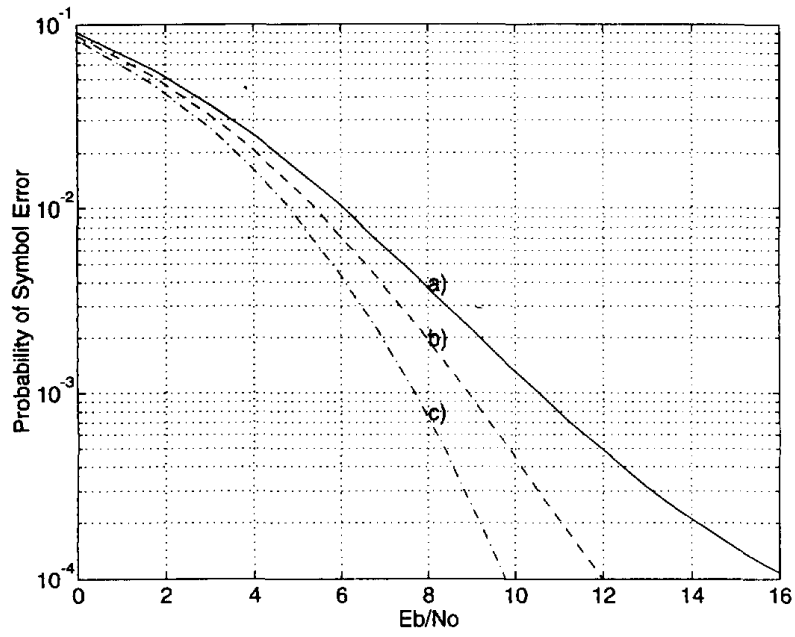

Fig. 9. Probability of symbol error for different pulse shapes and orthogonal sequences; $\Delta_{m}=.7, \beta=.5$ and $N_{u}=N=128$ : (a) raised cosine pulse and non-Sylvester Hadamard matrix, (b) raised cosine pulse and Sylvester matrix, (c) optimum pulse and Sylvester matrix.

However, for a given processing gain, an increase in the chip period results in an increase in the symbol period, hence a decrease in the data rate. We can maintain the original data rate by using a multicarrier signaling scheme. The overall transmitted signal is comprised of several different bit steams, each modulated by a separate subcarrier.

Increasing the chip period by a factor of $M$, the number of subcarriers, reduces the bandwidth of each of the subband signals by a factor of $M$ relative to the bandwidth of the original spread spectrum signal so that the overall bandwidth is approximately the same as that of the single-carrier signal, assuming a spacing of $1 / T_{c}$ for the carrier frequencies. Fig. 10 shows the block diagram of the transmitter for the $k^{\text {th }}$ user of a three-carrier DS CDMA system.

The received signal is

$$
r(t)=\sum_{i=1}^{N_{u}} \sum_{j=1}^{M} A_{i} s_{i}^{j}\left(t-\tau_{i}\right) \cos \left(\omega_{j} t+\theta_{i}\right)+n(t)
$$

where $s_{i}^{j}(t)=\sum_{n} c_{n}^{i j} h\left(t-n T_{c}\right)$ and $M$ is the number of different subcarriers. To compute the probability of bit error for a given data stream (subcarrier) we need to consider the interference from other data streams with the same carrier and interference from data streams using other carriers. For arbitrary carrier spacings the approach in [14] may be used to determine the variance of the multiple-access noise. For carrier spacings equal to the chip rate the noise variance can be solved using a more direct approach. If carriers are spaced at multiples of the chip rate, and if rectangular chip pulses are used, then all of the bit streams belonging to a single user do not interfere with one another due to the orthogonality (in time) of the carriers. For ease of implementation, each bit stream corresponding to a single transmitter may be multiplied by the same spreading code. However in general the spreading codes for the subcarriers may be chosen so as to minimize the peak amplitude of the signal.

\section{A. Time-Limited Chip Pulses}

First we consider the case of a rectangular chip pulse $h(t)$. We assume that the receiver demodulates the data in each stream independently. Assuming two users and reception of a particular bit stream, the variance of the out-of-band multiple-access interference at the integrator output, when the synchronization offset is uniformly distributed, can be shown to be $\frac{T_{c}^{2}}{\left(16 \pi^{2} i^{2}\right)} \mu_{\operatorname{cor}}(N)\left[1-\operatorname{sinc}\left(2 \Delta_{m} i\right)\right]$, where the frequency separation is $i / T_{c} \mathrm{~Hz}$. This compares to the value of $T_{c}^{2} \mu_{\text {cor }}(N) \Delta_{m}^{2} / 24$ for case where the two signals have the same carrier. For this multicarrier system we can show that the $\gamma$ for the $k^{\text {th }}$ user's $m^{\text {th }}$ bit stream, corresponding to that in (4), is

$$
\gamma=\frac{\mu_{\mathrm{cor}}(N)}{N^{2}}\left(\frac{\Delta_{m}^{2}}{3}+\frac{1}{2 \pi^{2}} \sum_{\substack{j=1 \\ j \neq m}}^{M} \nu_{j}\right)
$$

where $\nu_{j}=\left(1-\operatorname{sinc}\left[2 \Delta_{m}(m-j)\right]\right) \frac{1}{(m-j)^{2}}$.

The question arises as to what value of $M$ should be used. If chip pulses are not band-limited, we expect that as we increase the number of carriers, the in-band noise will decrease (since the chip period is increased by a factor of $M$ ) whereas the total interference from the other subbands will rise; hence there is a trade-off between interference from in-band signals and intersubcarrier (or out-of-band) interference. Also, the larger the $M$ the greater the complexity of the system. To observe the effect of $M$ on the interference, in Fig. 11 we have plotted the in-band interference, out-of-band interference, and the total interference for the case of a rectangular chip pulse, where the in-band and out-of-band interference are given by the first and second terms of (34) (after expanding), respectively. The curves have been suitably normalized, and plotted as a function of $M$ where $M$ is odd, and assuming $m=\left\lfloor\frac{M}{2}\right\rfloor+1$, that is, the $m^{\text {th }}$ band is the center band. To account for the increased chip period we have replaced $\Delta_{m}$ by $\frac{\Delta_{m}}{M}$ in (34) and have assumed $\Delta_{m}=1$. As $M$ increases the in-band interference decreases monotonically; on the other hand, the out-of-band interference increases to a maximum value and then decreases. The decrease in the out-of-band interference is a result of the fact that as the fractional synchronization offset decreases the subcarrier signals become orthogonal, even though the number of interfering bands increases. In this particular example a good choice for $M$ is approximately 5 since there is a negligible reduction in the total interference for larger $M$.

\section{B. Band-Limited Chip Pulses}

Let us now assume that the chip pulse is not rectangular and that it is band-limited. Consider a pair of passband CDMA signals, belonging to two different users, and corresponding to different subcarrier frequencies, $s_{i}^{j}\left(t+\tau_{i}\right) \cos \left(w_{j} t+\theta_{i}\right)$ and $s_{k}^{m}\left(t+\tau_{k}\right) \cos \left(w_{m} t+\theta_{k}\right)$. We will generalize to the case of $N_{u}$ users later. Assuming the base station correlator is receiving 


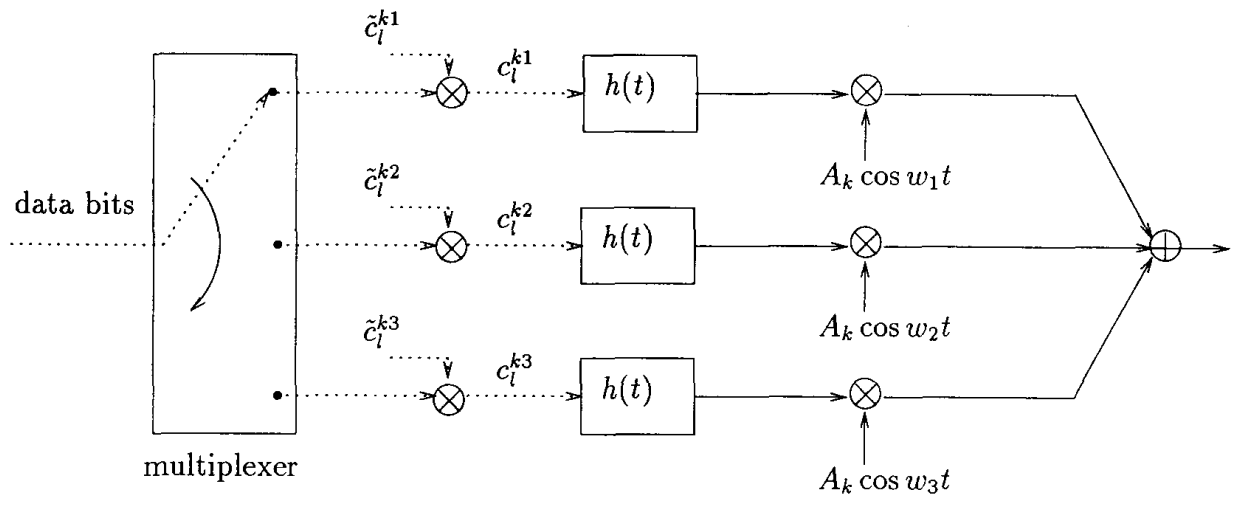

Fig. 10. Block diagram of a three-carrier DS CDMA transmitter.

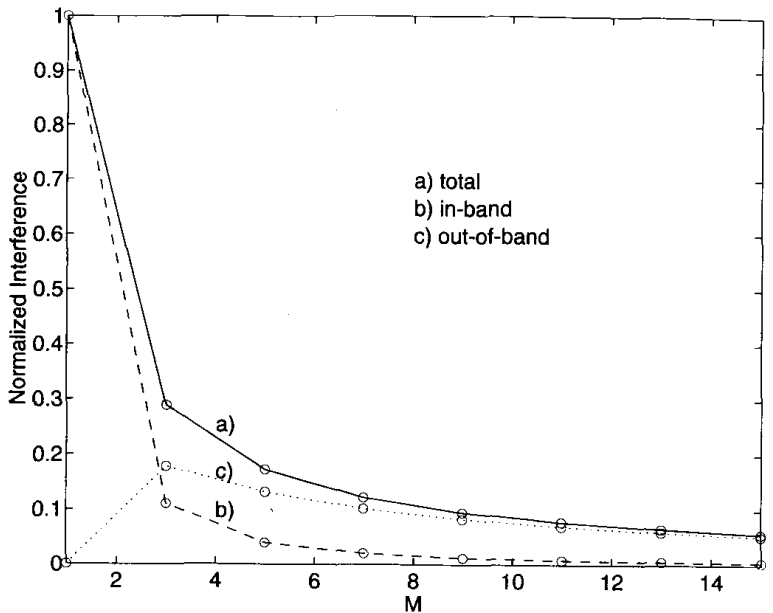

Fig. 11. Normalized in-band interference, out-of-band interference, and total interference as a function of the number of subcarriers $M$

$s_{k}^{m}(t)$ the variance of the multiple-access interference at the integrator output is

$$
\begin{aligned}
\sigma_{M}^{2}= & \mathcal{E}\left\{\psi^{2}\right\}=\frac{1}{4} \sum_{\substack{l=-\infty \\
l \neq 0}}^{\infty} \mu_{l} \mathcal{E} \\
& \cdot\left\{\left(\int_{-\infty}^{\infty} h\left(t+\tau-l T_{c}\right) h(t) \cos \left(w_{s} t+\theta\right) d t\right)^{2}\right\} .
\end{aligned}
$$

The expectation in (35) is with respect to $\theta$, modeled as uniform on $[0,2 \pi]$, and $\tau$.

Assuming that $h(t)$ has bandwidth equal to $\frac{1+\beta}{2 T_{c}}, 0 \leq \beta \leq 1$, we are interested only in the case where $w_{s}=\frac{2 \pi}{T_{c}}$ since the cross-correlation is 0 for $w_{s}=\frac{2 \pi k}{T_{c}}, k>1$. Assuming $f_{\Delta}(\cdot)$ and $h(\cdot)$ are even, it can be shown that each term in the summation in (35) is even with respect to $l$. Using Parseval's relation, the above can be put into the following frequency domain form

$$
\sigma_{M}^{2}=\frac{1}{4} \sum_{l=1}^{\infty} \mu_{l} \int_{-\Delta_{m}}^{\Delta_{m}} P(\Delta, l) f_{\Delta}(\Delta) d \Delta
$$

where

$$
P(\Delta, l)=\left|\int_{0}^{\frac{1+\beta}{2 T_{\mathrm{c}}}} H(f) H\left(f-\frac{1}{T_{c}}\right) \exp \left(j 2 \pi(l-\Delta) f T_{c}\right) d f\right|^{2} .
$$

Generalizing to the case of $N_{u}$ users, we assume the received signal is as in (33) and that the receiver is demodulating the data for the $m^{\text {th }}$ stream of the $k^{\text {th }}$ user, and that $\theta_{k}=\tau_{k}=0$. The integrator output is

$$
y \approx \pm \frac{A_{k} E_{s}}{2}+\psi_{\text {in }}+\psi_{\text {out }}+\eta
$$

where $\psi_{\text {in }}$ is the in-band multiple-access interference and $\psi_{\text {out }}$ is the out-of-band interference, due to the other $N_{u}-1$ users. Assuming that the $m^{\text {th }}$ band is an interior band, we can show that

$$
\gamma=\gamma_{1}+\frac{8 \sigma_{M}^{2} \sum_{\substack{i=1 \\ i \neq k}}^{N_{u}} A_{i}^{2}}{E_{s} \sum_{\substack{i=1 \\ i \neq 1 \\ i \neq k}}^{N_{u}} E_{i}} \approx \gamma_{1}+\frac{16 \sigma_{M}^{2}}{N^{2} E_{h}^{2}}
$$

where the rightmost expression in (38) was obtained assuming $E_{s} \approx N E_{h} . \gamma_{1}$ is the $\gamma$ value for the single-carrier scheme (obtained from Table I), and $\sigma_{M}^{2}$ is given by (36). Substituting (38) into (4) gives the bit error rate for the $k^{\text {th }}$ user's $m^{\text {th }}$ bit stream.

$\gamma$ is composed of two terms, the first resulting from the inband interference and the second arising from the out-of-band interference. In the design of the optimum pulse shape we minimized the first term. In a multicarrier system the criterion should be to minimize $\gamma$ in (38). However, since the first term can be much larger than the second, a criterion which minimizes the first should produce a pulse which is close to the optimal.

\section{System DEsign ASPECTS}

The multiple-access capability of a signaling scheme is an important system design issue. The allowable number of simultaneous users will depend on the background noise level $N_{0}$ which could be due to thermal noise or interference from other cells in the system, and on the required operating symbol error rate, $P_{e}$. For small $N_{0}$ and high $P_{e}$ the system will support $N$ users resulting in all codes being used. If it is not 
possible to support $N$ users then users (spreading sequences) should be dropped until the multiple-access interference is reduced to an acceptable level. In doing so the correlation matrix for all codes should be examined so that sequences resulting in large cross-correlations are dropped first. As an example the Sylvester matrix has one row which is equal to a cyclic shift (by one) of another row. One of these should be the first to be dropped.

Even after dropping some codes the probability of error for the users using the remaining codes will differ. This difference may be removed by having the users interchange their codes after each symbol in a manner similar to frequency hopping (i.e. "code hopping"). The algorithms for the hopping should be designed so as to achieve a maximum degree of randomization. Such algorithms will not significantly increase the complexity of the transmission scheme. Even with randomization and relatively stable clocks there will be some dependence between successive symbol errors if the variable $\Delta$ holds relatively constant from symbol to symbol. This effect could be removed by randomizing $\Delta$ every few periods of the spreading code.

\section{CONCLUSION}

A multicarrier orthogonal CDMA signaling scheme was introduced as a means to reduce interference in a multipoint-topoint CDMA network. Spreading codes and chip pulse shapes which minimize multiple-access interference in the case of quasi-synchronous operation were determined.

A sequence design criterion based on the mean square value of the sequence cross correlations (denoted as $\mu_{\text {cor }}(N)$ ) was used. For a given $N \times N$ Hadamard matrix, there is associated with it a value of $\mu_{\text {cor }}(N)$. The larger the $\mu_{\text {cor }}(N)$ value, the worse the performance of a quasi-synchronous CDMA system based on the given matrix. We searched for orthogonal matrices with small $\mu_{\text {cor }}(N)$ values. Sylvester-type Hadamard matrices were shown to have a $\mu_{\text {cor }}(N)$ value of approximately $\frac{2}{3} N$ for large $N$. It appears that for moderate to large $N$ most other Hadamard matrices have a $\mu_{\text {cor }}(N)$ value approximately equal to $N$.

Optimal chip pulses of various bandwidths that minimize multiple-access interference for quasi-synchronous systems were obtained. They were compared with the raised cosine pulses and were shown to have better performance at the higher excess bandwidths considered.

A multicarrier signaling scheme, designed to reduce chip level synchronization offsets between the users, was introduced. This quasi-synchronous CDMA scheme, based on orthogonal spreading codes and multicarrier modulation, is an alternative to an asynchronous scheme which utilizes sophisticated interference cancellation techniques.

\section{APPENDIX}

We derive (13). Defining $X_{i j}^{N}=\sum_{k=1}^{N} c_{k}^{i} c_{k+1}^{j}$, where $c_{k}^{i}$ is the $(i, k)^{\text {th }}$ element of an $N \times N$ Sylvester matrix, and

$$
\begin{aligned}
& c_{N+1}^{i}=c_{1}^{i}, \text { we use }(10) \text { to write } \\
& \begin{aligned}
N(N-1) \mu_{\text {cor }+}(N)= & \sum_{i=1}^{N} \sum_{\substack{j=1 \\
j \neq i}}^{N}\left(X_{i j}^{N}\right)^{2} \\
= & 8 \sum_{i=1}^{\frac{N}{2}} \sum_{\substack{j=1 \\
j \neq i}}^{\frac{N}{2}}\left(X_{i j}^{\frac{N}{2}}\right)^{2}-16 \sum_{i=1}^{\frac{N}{2}} \sum_{\substack{j=1 \\
j \neq i}}^{\frac{N}{2}}\left(X_{i j}^{\frac{N}{2}}\right) c_{\frac{N}{2}}^{i} \\
& +16\left(\frac{N}{2}\right)\left(\frac{N}{2}-1\right)
\end{aligned}
\end{aligned}
$$

where (A.1) follows because $X_{i j}^{N}$ equals $2 X_{i j}^{\frac{N}{2}}$ if $i$ and $j \epsilon\left\{1 \cdots \frac{N}{2}\right\}, 0$ if $i \epsilon\left\{1 \cdots \frac{N}{2}\right\}$ and $j \epsilon\left\{\frac{N}{2}+1 \cdots N\right\}$, or $2 X_{i-\frac{N}{2}, j-\frac{N}{2}}^{\frac{N}{2}}-4 c_{\frac{N}{2}}^{i-\frac{N}{2}}$ if $i$ and $j \epsilon\left\{\frac{N}{2}+1 \cdots N\right\}$. We also note that

$$
\begin{aligned}
\sum_{i=1}^{\frac{N}{2}} \sum_{\substack{j=1 \\
j \neq i}}^{\frac{N}{2}}\left(X_{i j}^{\frac{N}{2}}\right) c_{\frac{N}{2}}^{i}= & \sum_{i=1}^{\frac{N}{4}} \sum_{\substack{j=1 \\
j \neq i}}^{\frac{N}{4}}\left(X_{i j}^{\frac{N}{2}}\right) c_{\frac{N}{2}}^{i} \\
& +\sum_{i=\frac{N}{4}+1}^{\frac{N}{2}} \sum_{\substack{j=\frac{N}{4}+1 \\
j \neq i}}^{\frac{N}{2}}\left(X_{i j}^{\frac{N}{2}}\right) c_{\frac{N}{2}}^{i} \\
= & 2 \sum_{i=1}^{\frac{N}{4}} \sum_{\substack{j=1 \\
j \neq i}}^{\frac{N}{4}}\left(X_{i j}^{\frac{N}{4}}\right) c_{\frac{N}{2}}^{i} \\
& +\sum_{\substack{i=1 \\
\frac{N}{4}}}^{\frac{N}{4}}\left(2 X_{\substack{j=1 \\
j \neq i}}^{\frac{N}{4}}-4 c_{\frac{N}{4}}^{i}\right) c_{\frac{N}{2}}^{i+\frac{N}{4}} \\
= & 4\left(\frac{N}{4}\right)\left(\frac{N}{4}-1\right)
\end{aligned}
$$

since $c_{\frac{N}{2}}^{i+\frac{N}{4}}=-c_{\frac{N}{4}}^{i}=-c_{\frac{N}{2}}^{i}$ for $i \epsilon\left\{1 \ldots \frac{N}{4}\right\}$. (A.1) then reduces to

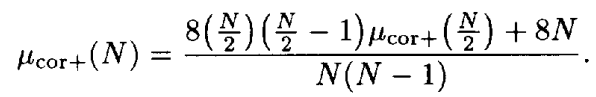

This is a recursive formula for $\mu_{\text {cor }+}(N)$, which may be interpreted as a first-order difference equation and solved using the initial condition $\mu_{\text {cor }+}(2)=0$ to obtain

$$
\mu_{\text {cor }+}(N)=\frac{2 N^{2}-8}{3(N-1)}
$$

In a similar fashion, we can show that

$$
\mu_{\text {cor }-}(N)=\frac{2 N^{2}+4}{3(N-1)} \text {. }
$$

Equation (13) then follows from (A.4), (A.5), and (9).

\section{REFERENCES}

[1] K. S. Gilhousen, I. M. Jacobs, R. Padovani and L. A. Weaver, "Increased capacity using CDMA for mobile satellite communication," IEEE $J$. Select. Areas in Commun., vol. JSAC-8, pp. 503-514, May 1990.

[2] R. L. Pickholtz, L. B. Milstein and D. L. Schilling, "Spread-spectrum for mobile communications," IEEE Trans. Veh. Techn., vol. VT-40, pp. 313-321, May 1991. 
[3] J. K. Omura and P. T. Yang, "Spread-spectrum S-CDMA for personal communications services," Proc. IEEE Milcom., San Diego, CA, Oct. 1992, pp. 269-273.

[4] M. B. Pursley, "Performance evaluation for phase-coded spread-spectrum multiple-access communications-Part I: System analysis," IEEE Trans. Commun., vol. COM-25, pp. 795-799, Aug. 1977

[5] M. K. Varanasi and B. Aazang, "Multistage detection in asynchronous code-division multiple-access communications," IEEE Trans. Commun., vol. COM-38, pp. 509-519, Apr. 1990.

[6] K. S. Gilhousen, I. M. Jacobs, R. Padovani, A. J. Viterbi, L. A. Weaver and C. E. Wheatley, "On the capacity of a cellular CDMA system," IEEE Trans. Veh. Techn., vol. VT-40, pp. 303-312, May 1991.

[7] G. E. Bottomley, "Signature sequence selection in a CDMA system with orthogonal coding," IEEE Trans. Veh. Techn., vol. 42, pp. 62-68, Feb. 1993.

[8] D. T. Magill and T. G. Kirke, "Optimal short-range CDMA codes of length 16 for wireless communications," Proc. ICWC'92, pp. 195-198.

[9] H. D. Schotten and M. Antweiler, "Iterative construction of sequences with low cross-correlation values," Proc. ICC 93, Geneva, Switzerland, pp. $156-160$.

[10] R. De Gaudenzi, C. Elia and R. Viola, "Bandlimited quasi-synchronous CDMA: A novel satellite access technique for mobile and personal communication systems," IEEE J. Select. Areas in Commun., vol. 10, pp. 328-342, Feb. 1992.

[11] E. S. Sousa, "Interference modeling in direct-sequence spread-spectrum packet radio networks," IEEE Trans. Commun., vol. COM-38, pp. 1475-1482, Sept. 1990

[12] F. J. MacWilliams and N. J. A. Sloane, The Theory of Error-Correcting Codes, North Holland, Amsterdam, 1986

[13] E. S. Sousa and S. Pasupathy, "Pulse shape design for teletext data transmission," IEEE Trans. Commun., vol. COM-31, No. 7, pp. 871-878, July 1993.

[14] E. S. Sousa, "The performance of a direct sequence spread spectrum CDMA system utilizing unequal carrier frequencies," Proc. IEEE 2 nd Int. Symp. on Spread Spectrum Techniques and Applications, Yokohama, Japan, Dec. 1992.

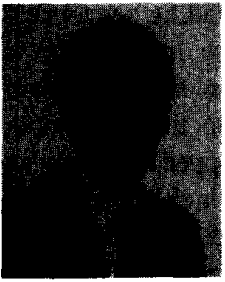

Victor DaSilva (S'94) received the B.A.Sc. degree in engineering science and the M.A.Sc. degree in electrical engineering from the University of Toronto in 1992 and 1993, respectively. He is currently working towards the Ph.D. degree at the Department of Electrical and Computer Engineering at the University of Toronto, where he is also a research and teaching assistant. He holds a Natural Sciences and Engineering Research Council of Canada (NSERC) Postgraduate Scholarship.

$\mathrm{He}$ has worked on several projects for Bell Mobility Cellular, including a study on dynamic channel allocation strategies. His research interests are in the areas of indoor wireless and mobile communication systems.

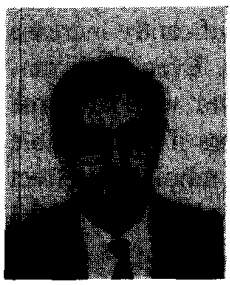

Elvino S. Sousa (S ${ }^{\circ} 80-M^{`} 86$ ) was born in Graciosa Azores, Portugal on December 28, 1956. He received the B.A.Sc. degree in engineering science, and the M.A.Sc. degree in electrical engineering from the University of Toronto, Canada, in 1980 and 1982, respectively, and the Ph.D. degree in electrical engineering from the University of Southern California, USA, in 1985.

Since 1986, he has been with the Department of Electrical and Computer Engineering at the University of Toronto where he is presently an Associate Professor. Since 1986, he has been a Natural Sciences and Engineering Research Council of Canada (NSERC) University Research Fellow. He has performed research in the areas of packet radio networks, spread spectrum systems, mobile communications, and indoor wireless communications. His research interests are in the area of mobile and personal communication networks. At the University of Toronto, he has taught graduate courses in error-correcting codes and mobile communications. 